DOI: 10.12957/demetra.2015.16712

\title{
Autoavaliação e fatores associados ao consumo de frutas e hortaliças em adultos de Brasília
}

\section{Self-assessment and factors associated with fruit and vegetable consumption in adults from Brasília, Brazil}

Clíslian Luzia da Silva'

Teresa Helena Macedo da Costa'

Eduardo Freitas da Silva ${ }^{2}$

' Universidade de Brasília, Faculdade de Ciências da Saúde, Departamento de Nutrição. BrasíliaDF, Brasil.

2 Universidade de Brasília, Instituto de Ciências Exatas, Departamento de Estatística. Brasília-DF, Brasil.

Financiamento: Parte da pesquisa foi financiada com recursos do CNPq (Processo $\mathrm{n}^{0}$ 474.665/2004-6) e taxa de bancada (Processo $\left.n^{0} 302.520 / 2008-2\right)$.

Correspondência / Correspondence

Clíslian Luzia da Silva

E-mail: clislian@gmail.com

\section{Resumo}

Objetivou-se comparar a autoavaliação de consumo de frutas e hortaliças com a recomendação do guia alimentar da população brasileira e avaliar as características associadas em indivíduos residentes emBrasília, Distrito Federal. Uma amostra por conglomerados foi utilizada para selecionar 98 indivíduos que foram entrevistados por telefone. Obtiveram-se dados de frequência, porção e autoavaliação da ingestão de frutas e hortaliças. Razões de prevalência (RP) foram calculadas por regressão de Poisson múltipla com variância robusta. A concordância entre a percepção individual do consumo e a avaliação técnica foi obtida pelo coeficiente kappa simples. A maioria dos indivíduos consome menos que trêsporções diárias de frutas (68\%) e hortaliças (77\%). A frequência de ingestão de frutas e hortaliças em cincoou mais dias da semana foi de $69,5 \%$ e $81,5 \%$, respectivamente. No modelo, cada aumento de um ano de estudo apresentou associação positiva com o aumento de $36 \%$ na adequação do consumo de frutas e hortaliças ( $\mathrm{p}<0,01$; IC 1,17 a 1,59). A concordância entre aautoavaliação e a avaliação técnica do consumo de frutas e de hortaliças revelou-se fraca. Nesse contexto os programas de incentivo ao consumo de frutas e hortaliças devem informar a quantidade e também o tamanho da porção.

Palavras-chave: Porção. Ingestão. Vegetais. Frutas. Consumo de Alimentos. Recomendações Nutricionais. 


\section{Abstract}

This study aimed to compare the self-assessment intake of fruits and vegetables with the Brazilian's Food Guide recommendation and evaluate associated characteristics in subjects living in Brasilia, Federal District. Individuals were selected from a random cluster sample of residences and a total of 98 adults were interviewed by telephone. Frequency of intake, portions and self-assessment intake of fruits and vegetables were obtained. Prevalence ratio (PR) was calculated with a multiple Poisson regression with robust variance. The agreement between selfassessment intake and technical evaluation was performed using simple kappa coefficient. Majority of subjects consume less than three portions of fruits (68\%) and vegetables (77\%) daily. The frequency of intake of fruits and vegetables on five or more days of the week were $69.5 \%$ and $81.5 \%$, respectively. In the model each one year of study was positively associated with a $36 \%$ increase in adequacy of fruits and vegetables consumption ( $p<0.01$; CI 1.17 a 1.59). The agreement between self- assessment and technical evaluation of fruits and vegetables intake presented low values. In this context programs that encourage consumption of fruits and vegetables must report the amount as well as the portion size.

Key words: Portion Size. Consumption. Vegetable. Fruit. Food Consumption. Recommended Dietary Allowances.

\section{Introdução}

O consumo mínimo de $400 \mathrm{~g}$ de frutas e hortaliças diariamente é recomendado pela Organização Mundial da Saúde (OMS) para prevenir as doenças crônicas não transmissíveis. Programas de incentivo ao consumo destes alimentos foram elaborados principalmente em países desenvolvidos, sendo denominados programas do tipo "Five A Day", em que são sugeridas cincoporções diárias de frutas e hortaliças. ${ }^{2}$ No Brasil, foi desenvolvido um guia alimentar que recomenda o consumo mínimo de seisporções ao dia, divididas igualmente para frutas e hortaliças. ${ }^{3}$

Apesar das recomendações, a maioria da população não consome quantidades adequadas de frutas e hortaliças. De acordo com a Pesquisa de Vigilância de Fatores de Risco e Proteção para Doenças Crônicas por Inquérito Telefônico (Vigitel), ${ }^{4}$ apenas 20\% (IC95\% 19-21) dos brasileiros consomem cincoou mais porções de frutas e hortaliças em cinco ou mais dias da semana.Dentre as 
capitais dos estados brasileiros, o Distrito Federal (DF) destaca-se por conter maior proporção de adultos com ingestão de cincoou mais porções destes alimentosem cinco ou mais dias na semana. Mesmo assim, verifica-se que apenas 24\%(IC95\% 20,8-27,3) da população do DF consomem quantidades adequadas. ${ }^{4}$

Alguns estudos identificam a presença de características importantes que podem favorecer ou prejudicar o consumo destes alimentos. A ingestão de frutas e hortaliças tem sido positivamente relacionadaà pratica de atividade física, ${ }^{5-9}$ renda, ${ }^{6,9-11}$ idade e nível de escolaridade ${ }^{4,11,12} \mathrm{e}$ inversamente relacionada ao hábito de fumar, ${ }^{6-9}$ Índice de Massa Corporal (IMC) ${ }^{5,6,13,14}$ e densidade energética da dieta. ${ }^{13}$ Além disso, observa-se também que o sexo pode influenciar no consumo destes alimentos, já que mulheres tendem a consumir mais frutas e hortaliças do que homens. ${ }^{4,9,11}$

O conhecimento dos benefícios relacionados à ingestão de frutas e hortaliças é um fator motivacional para aumentar o seu consumo. ${ }^{11,15-18} \mathrm{Em}$ publicação recente sobre as barreiras e facilitadores para o consumo destes alimentos na população de Brasília, descrevemos que os principais fatores motivacionais mencionados pelos brasilienses para o consumo de frutas e hortaliças foi vinculada à saúde e ao sabor agradável. $\mathrm{O}$ aspecto ligado à saúde foi identificado em $52 \%$ das respostas para frutas e em $57 \%$ das respostas para hortaliças. Enquanto,o sabor desagradável para hortaliças (36\% das respostas) e a falta hábito para as frutas (23\% das respostas) são as principais barreiras mencionadas. ${ }^{19}$

A OMS orienta que as iniciativas que promovem o consumo de frutas e hortaliças devem ser baseadas em evidências científicas que enfatizem os benefícios destes alimentos para a saúde. ${ }^{20}$ Apesar disso, essas mensagens podem não ser eficientes em pessoas que acreditam possuir consumo adequado de frutas e hortaliças. ${ }^{11,21-24}$ Por isso, para que as estratégias que promovam a ingestão de tais alimentos sejam eficientes, além da identificação dos grupos de pessoas que consomem abaixo do recomendado e das dificuldades encontradas para aumentar este consumo, é necessário também conhecer a quantidade de frutas e hortaliças que os indivíduos consideram ideal para o consumo.

Os objetivos deste estudo são comparar as características e autoavaliação do consumo entre indivíduos adultos residentes em Brasíliaque atingem ou não a recomendação do Guia Alimentar para a população Brasileira de consumo diário de três porções de frutas e detrêsporções de hortaliças; avaliar associações entre o consumo adequado de frutas e hortaliças com as características sociodemográficase a classificação do estado nutricional nesta população.As hipóteses do estudo são que o consumo de frutas e hortaliças está associado ao sexo, IMC e escolaridade, e que existe baixa concordância entre o consumo de frutas e hortaliças e a autoavaliação. 


\section{Métodos}

Trata-se de um estudo descritivo, com abordagem quantitativa. O estudo qualitativo desenvolvido com essa amostra foi publicado anteriormente, assim como também a descrição da amostragem e dos critérios de inclusão e exclusão. ${ }^{19}$ Resumidamente, uma amostra aleatória por conglomerados de domicílios foi selecionada a partir da lista de endereços residenciais da Companhia Energética de Brasília, que abrange 100\% dos domicílios. Os conglomerados foram formados pelas regiões administrativas que compõem a cidade de Brasília: Asa Sul, Asa Norte, Vila Planalto e Setor Militar Urbano. A amostra de 250 residências foi calculada do total de 82.680domicílios, mantendo-se erro alfa em $5 \%$ e considerou-se a prevalência de $80 \%$ de inatividade física, que era o desfecho principal da pesquisa de base que deu origemà amostra do estudo. ${ }^{25} \mathrm{~A}$ amostra foi representativa da população da cidade de Brasília. Como critérios de seleção, foram entrevistados todos os moradores adultos e que concordaram em participar, sendo considerados moradores todas as pessoas que dormiam no domicílio, com exceção de visitantes. Participaram da pesquisa apenas os indivíduos com 20 anos ou mais a fim de evitar a inclusão de algum sujeito com maturação tardia, principalmente no sexo masculino.

Para participação no presente estudo, foram incluídas, de modo sistemático e mantendo-se a aleatoriedade amostral, $50 \%$ das 250 residências $(n=124)$. As entrevistas foram do tipo estruturada e realizadas por telefone com um morador de cada domicílio, identificado como número 1 no protocolo de Thomaz et al. ${ }^{25}$ As ligações ocorreram no Laboratório de Bioquímica da Nutrição, Núcleo de Nutrição, da Universidade de Brasília (UnB) e foram gravadas.

O consentimento livre e esclarecido do entrevistado para a participação na pesquisa foi gravado e os procedimentos metodológicos foram preparados com base nos regulamentos éticos e científicos do Conselho Nacional de Saúde do Ministério da Saúde. O projeto foi aprovado pelo Comitê de Ética em Pesquisa com Seres Humanos da Faculdade de Ciências da Saúde da Universidade de Brasília (registro 101/2006).

As informações sociodemográficas e de estatura foram retiradas do protocolo do estudo de Thomaz et $\mathrm{al}^{25}$, que foi realizado presencialmente um ano antes no domicílio. O peso atual foi informado na ligação telefônica. Na população de Brasília determinamos a validade do peso, altura e IMC autorreferidos, sendo adequada a utilização dessa informação para acompanhamento da população com a devida precisão. ${ }^{26}$ A partir do peso e da altura foi calculado o IMC [peso (Kg)/ estatura $(m)^{2}$ ], e o estado nutricional foi estabelecido utilizando-se a classificação da $\mathrm{OMS}^{20}$ para adultos e de Lipschitz ${ }^{27}$ para idosos.

A informação da finalização do maior nível de escolaridade foi obtida e posteriormente convertida em anos de estudo. Foram computados 11 anos de estudo para a conclusão do ensino fundamental, 11 a 14 anos para o ensino médio e 15 anos ou mais para a conclusão do nível superior. 
Foi utilizado um formulário contendo perguntas referentesàfrequência e porção de consumo de frutas e hortaliças e autoavaliação do consumo. Ascaracterísticas de consumo foram obtidas separadamente para frutas e para hortaliças. Nas questões sobre o consumo de frutas, foi perguntado o número de frutas ou fatias ingeridas por dia. Uma unidade de fruta ou fatia foi considerada como uma porção. Para hortaliças, questionou-se o número de colheres de sopa consumidas em cada dia considerando-se umaporção igual a três colheres de sopa. Neste último caso, para calcular o número de porções consumidas de hortaliças, dividiu-se a quantidade de colheres de sopa informadas pelo entrevistado portrês. Para se definir o número de porções de frutas e hortaliças consumidas diariamente, considerou-se:

(Número de porções consumidas x frequência semanal de consumo)/7

No processo de análise dos dados, o consumo de frutas e hortaliças foi avaliado separadamente e em conjunto. No primeiro caso, avaliou-se o consumo considerando, como referência, três porções diárias para frutas e trêspara hortaliças. Para análise em conjunto, somou-se o número de porções de frutas e hortaliças ingeridas diariamente por cada indivíduo e considerou-se como ingestão adequadaseisou mais porções de acordo com as recomendações do Guia Alimentar da População Brasileira. ${ }^{3}$

Na análise de autoavaliação do indivíduo sobre a sua ingestão atual de frutas e de hortaliças, os sujeitos tinham como opção de resposta: baixo, adequado, elevado ou não sabe responder sobre o consumo. Desse modo, sujeitos que responderam acreditar que a sua ingestão estava baixa ou não souberam informar, tiveram as suas respostas classificadas no grupo de autoavaliaçãode inadequação. Para os entrevistados que acreditavam que o seu consumo estava adequado ou elevado, as respostas foram incluídas na categoria de autoavaliaçãode adequação.

Com relação à frequência de consumo, considerou-se consumo regular de frutas e de hortaliças a ingestão destes alimentos em pelo menos cincodias da semana, conforme protocolo utilizado na Pesquisa sobre Vigilância de Fatores de Risco e Proteção para Doenças Crônicas por Inquérito Telefônico (Vigitel). ${ }^{4}$

Para a realização da análise descritiva, foram calculados média, desvio padrão e proporções. Uma análise de regressão de Poisson com o procedimento de variância robusta foi utilizada, incorporando-se os pesos amostrais (inversos das probabilidades de inclusão), o efeito do conglomerado e a estratificação. ${ }^{28}$ A adequação do consumo de frutas e hortaliças foi considerada a variável dependente do estudo. As variáveis independentes presentes no modelo foram: sexo, idade, número de horas trabalhadas por semana, classe sócio econômica (Classe A, Classes B, C, D e E, de acordo com o critério de Classificação Econômica Brasil ${ }^{29}$ ), estado civil (Solteiro, Casado, Viúvo/Divorciado), anos de estudo e IMC $\left(\mathrm{Kg} / \mathrm{m}^{2}\right)$. As interações entre as variáveis independentes foram testadas e nenhuma revelou significância estatística. A multicolinearidade entre as variáveis 
foi avaliada e seu efeito não foi detectado. As razões de prevalência foram calculadas a partir dos resultados do modelo e o nível de significância adotado foi $\mathrm{p}<0,05$.

Foi avaliada a concordância entre a autoavaliação do consumo de frutas e de hortaliças e a avaliação técnica sendo utilizado como medida de concordância, o coeficiente kappasimples. ${ }^{30} \mathrm{~A}$ análise dos dados foi realizada utilizando-se os programas SAS v.9.2 e STATA v.10.

\section{Resultados}

Participaram da pesquisa 98 indivíduos. De acordo com Tabela 1, podem-se observar as característicassociodemográficas e antropométricas dos sujeitos na amostra total e separados segundo adequação do consumo de frutas e hortaliças. Verifica-se que há semelhança na proporção entre homens e mulheres $(52 \%, 48 \%$, respectivamente) bem como entre indivíduos eutróficos e sobrepesos/obesos (52,48\%, respectivamente). A média de idade é de aproximadamente 46 anos ( \pm $15,4)$ e há maior predominância da faixa etária de 20 a 59 anos $(84 \%)$. A maioria dos entrevistados são casados (59\%), e possuem 15 ou mais anos de estudo (55\%). No que diz respeito à carga horária de trabalho, há maior concentração de sujeitos que trabalham 40 horas semanais ou mais (52\%).

Analisando-se os sujeitos segundo adequação do consumo de frutas e hortaliças, verificouse que a maioria $(83 \%, n=81)$ não atinge a recomendação do consumo de frutas e hortaliças conforme proposto peloGuia Alimentar para População Brasileira ${ }^{3}$ (Tabela 1). Sabendo-se que tais alimentos devem compor de 9 a 12\% do valor energético total (VET) e considerando-se uma dieta de $2000 \mathrm{kcal}$, dentre os sujeitos com consumo inadequado, o valor calórico médio ingerido de frutas e hortaliças equivale a $120 \mathrm{kcal}( \pm 66,4)$, correspondendo a apenas $6 \%$ do VET.

Tabela 1. Características sociodemográficas e classificação do estado nutricional de adultos de Brasília, Distrito Federal, segundo adequação no consumo de frutas/hortaliças - 2007.

\begin{tabular}{|c|c|c|c|}
\hline VARIÁVEL & $\begin{array}{l}\text { Inadequado }^{\mathrm{a}} \\
\quad(\mathrm{N}=81)\end{array}$ & $\begin{array}{c}\text { Adequado }^{\mathrm{b}} \\
(\mathrm{N}=17)\end{array}$ & $\begin{array}{c}\text { Total } \\
(\mathrm{N}=98)\end{array}$ \\
\hline & $\mathrm{n}^{\underline{\underline{o}}}$ & no & $\mathrm{n}^{\underline{\underline{o}}}$ \\
\hline
\end{tabular}

SEXO

$\begin{array}{lllllll}\text { Masculino } & 42 & 52 & 9 & 53 & 51 & 52 \\ \text { Feminino } & 39 & 48 & 8 & 47 & 47 & 48\end{array}$




\begin{tabular}{|c|c|c|c|}
\hline VARIÁVEL & $\begin{array}{l}\text { Inadequado }^{\mathrm{a}} \\
\qquad(\mathrm{N}=81)\end{array}$ & $\begin{array}{l}\text { Adequado }^{\mathrm{b}} \\
(\mathrm{N}=17)\end{array}$ & $\begin{array}{c}\text { Total } \\
(\mathrm{N}=98)\end{array}$ \\
\hline
\end{tabular}

FAIXA ETÁRIA (anos)

$\begin{array}{lcccccc}20-39 & 32 & 40 & 3 & 18 & 35 & 36 \\ 40-59 & 35 & 43 & 12 & 71 & 47 & 48 \\ >=60 & 14 & 17 & 2 & 12 & 16 & 16\end{array}$

ESTADO CIVIL

Solteiro

20

25

3

18

23

23

Casado

49

60

9

53

58

59

Separado/ Viúvo

12

15

5

29

17

17

ANOS DE ESTUDO

$<11$

11

14

0

0

11

11

11-14

30

37

3

18

33

34

$>=15$

40

49

14

82

54

55

CLASSE SOCIAL

$\begin{array}{lcccccc}\text { A e B } & 64 & 79 & 17 & 100 & 81 & 83 \\ \text { C, D e E } & 17 & 21 & 0 & 0 & 17 & 17\end{array}$

TRABALHO (horas/sem)

$\begin{array}{lllllll}0 \text { a } 19 & 17 & 21 & 7 & 41 & 24 & 24 \\ 20 \text { a } 39 & 20 & 25 & 3 & 18 & 23 & 23 \\ >\text { ou }=40 & 44 & 54 & 7 & 41 & 51 & 52\end{array}$

ESTADO NUTRICIONAL

\begin{tabular}{lllllll} 
Eutróficos & 43 & 53 & 8 & 47 & 51 & 52 \\
Sobrepesos/obesos & 38 & 47 & 9 & 53 & 47 & 48 \\
\hline
\end{tabular}

a: Consomem menos que seis porções de frutas e hortaliças por dia

b: Consomemseis ou mais porções de frutas e hortaliças por dia 
De acordo com a Tabela 2, verifica-se que as variáveis sexo, idade, horas de trabalho, classe social, estado civil e IMC não se mostraram associadas com a adequação ao consumo de frutas e hortaliças. Entretanto, o número de anos de estudos está diretamente associado ao consumo destes alimentos sendo que, para cada aumento de um ano de estudo a prevalência de adequação da ingestão de frutas e hortaliças aumenta em $36 \%$.

Tabela 2. Razões de prevalência e associação da adequação de consumo de frutas/hortaliças com as características sociodemográficas e classificação do estado nutricional de adultos de Brasília, Distrito Federal - 2007.

\begin{tabular}{lccc}
\hline VARIÁVEL & RPa & IC de $95 \%^{\mathrm{b}}$ & Valor de $\mathrm{p}$ \\
SEXO & & & 0,21 \\
$\quad$ Masculino x Feminino & 1,62 & 0,76 a 3,47 & 0,75 \\
IDADE & 1,01 & 0,96 a 1,06 & \\
ESTADO CIVIL & & & 0,24 \\
$\quad$ Divorciado/Viúvo x Casado & 1,86 & 0,65 a 5,30 & 0,37 \\
$\quad$ Solteiro x Casado & 1,62 & 0,55 a 4,76 & 0,40 \\
CLASSE SOCIAL & & & 0,25 \\
$\quad$ A x B,C,D e E & 1,60 & 0,53 a 4,82 & 0,16 \\
TRABALHO (horas/sem) & 0,98 & 0,94 a 1,01 & $<0,01$ \\
ESTADO NUTRICIONAL (IMC) & 0,94 & 0,87 a 1,02 & \\
ANOS DE ESTUDO & 1,36 & 1,17 a 1,59 & \\
\hline
\end{tabular}

aRazão de prevalência

${ }^{\mathrm{b}}$ Intervalo de confiança

Ao avaliarmos a concordância entre a autoavaliação do consumo de frutas e de hortaliças com a avaliação técnica deste consumo (Tabela 3), verifica-se que o valor de Kappa e o intervalo de confiança (IC) de 95\% foi maior para as frutas do que para as hortaliças (Kappa: 0,41; IC 95\% 0,22 - 0,59; Kappa: 0,24; IC 95\% de 0,09 - 0,39, respectivamente). Sabendo-se que a concordância é substancial entre valores de Kappa de 0,60 a 0,79, moderada entre 0,40 a 0,59 e fraca $<0,40$, inferese que a concordância teve variação de nível moderado para frutas e nível fraco para hortaliças. Observa-se ainda que quase metade dos indivíduos com consumo inadequado de hortaliças 
considera-o adequado (47\%), enquanto $27 \%$ com ingestão de frutas abaixo do recomendado acreditam possuir consumo adequado destes alimentos.

Considerando-se o padrão de consumo de frutas e hortaliças, observam-se baixas proporções de indivíduos com consumo adequado (31,5\% n=31, 23,5\% $\mathrm{n}=23$, respectivamente), enquanto porcentagem elevada possui frequência de ingestão destes alimentos em cincoou mais dias da semana $(69,5 \% \mathrm{n}=68$ e $81,5 \% \mathrm{n}=80$, respectivamente).

Tabela 3. Autoavaliação do consumo segundo adequação de consumo de frutas e hortaliças entre adultos de Brasília, Distrito Federal - 2007.

\begin{tabular}{|c|c|c|c|c|c|c|c|c|}
\hline \multirow{3}{*}{$\begin{array}{c}\text { Autoavaliação do } \\
\text { consumo }\end{array}$} & \multicolumn{4}{|c|}{ Frutas } & \multicolumn{4}{|c|}{ Hortaliças } \\
\hline & \multicolumn{2}{|c|}{$<3$ porções } & \multicolumn{2}{|c|}{$>=3$ porções } & \multicolumn{2}{|c|}{$<3$ Porções } & \multicolumn{2}{|c|}{$>=3$ porções } \\
\hline & $\mathrm{N}$ & $\%$ & $\mathrm{~N}$ & $\%$ & $\mathrm{~N}$ & $\%$ & $\mathrm{~N}$ & $\%$ \\
\hline Adequado & 18 & 27 & 22 & 71 & 35 & 47 & 19 & 83 \\
\hline Inadequado & 49 & 73 & 9 & 29 & 40 & 53 & 4 & 17 \\
\hline Карра & \multicolumn{4}{|c|}{0,41} & \multicolumn{4}{|c|}{0,24} \\
\hline IC $95 \%$ & \multicolumn{4}{|c|}{$0,23-0,59$} & \multicolumn{4}{|c|}{$0,09-0,39$} \\
\hline
\end{tabular}

\section{Discussão}

Este estudo identificou proporção elevada de indivíduos com ingestão insuficiente de frutas e hortaliças. Resultado semelhante foi encontrado na população brasileira no ano de 2011, em estudo de Vigilância dos Fatores de Risco e Proteção para Doenças Crônicas por Inquérito Telefônico - Vigitel. ${ }^{4}$ Além disso, foi observado que dentre os sujeitos com consumo inadequado, seria necessário dobrar a quantidade da ingestão de frutas e hortaliças a fim de alcançar o valor recomendado pelo Guia Alimentar de três porções diárias de cada alimento. ${ }^{3}$

A porcentagem de indivíduos com consumo inadequado de frutas e hortaliças encontrada no presente estudo está acima do relatado em outros países. Tal proporção está $20 \%$ acima do encontrado em estudo de Ashfield-Watt $e^{2}{ }^{31}{ }^{31}$ realizado com ingleses, enquanto que, em amostra composta por 10 países europeus, verificou-se que oitodeles apresentaram proporções menores de sujeitos com consumo inadequado de frutas e hortaliças, cujas porcentagens variaram entre $37 \%$ a $78 \% .^{32}$ 
Vale ressaltar que as diferenças entre os resultados podem ser explicadas pelas recomendações dos guias alimentares e a descrição do grupo de frutas e hortaliças em cada país. Enquanto em países da Europa são recomendadas cincoporções, o Guia Alimentar para a População Brasileira preconiza consumo de seisporções diárias destes alimentos, tendo por base uma dieta de 2000kcal e $12 \%$ da energia da dieta proveniente de frutas e hortaliças. ${ }^{3}$ Sendo assim, o valor total recomendado pelo Guia é de $570 \mathrm{~g} / \mathrm{dia}$, valor que ultrapassa em aproximadamente $40 \%$ a recomendação da OMS ${ }^{1}$ e classifica um maior número de indivíduos com consumo inadequado. Além disso, diferente do Brasil, em outros países há inclusão de sucos no que se refere às frutas bem como de tubérculos e raízes nas porções de hortaliças, fato que favorece a discrepância.

Dentre as variáveis sociodemográficas pesquisadas, apenas anos de estudo mostrou-se positivamente associada ao consumo de frutas e hortaliças. Foi observado que, para cada aumento de um ano de estudo, a prevalência de adequação à ingestão de fruta e hortaliça aumenta substancialmente. Resultados semelhantes também foram encontrados na literatura. ${ }^{33-35}$ De fato, há evidências de que o aumento da escolaridade aumenta a preocupação sobre o efeito da dieta na manifestação de doenças, ${ }^{36}$ bem como a consciência sobre a necessidade de mudanças alimentares e sobre a qualidade da dieta ingerida, ${ }^{21}$ permitindo que indivíduos mais esclarecidos possuam um padrão alimentar mais saudável. ${ }^{37}$

Não foi encontrada associação entre IMC e consumo de frutas e hortaliças. Tal resultado concorda com o estudo de Neutzlinget $a l^{9}$ no qual foi pesquisado adultos de uma cidade do Sul do Brasil. Estudos que encontram associação entre essas variáveis são normalmente longitudinais e relacionam o consumo de frutas e hortaliças à proteção contra ganho de peso. ${ }^{38,39}$

Não foram encontrados estudos que analisam o número de porções consumidas de frutas e de hortaliças separadamente na população adulta do DF. Logo, esta é a primeira pesquisa que apresenta os resultados com esse detalhamento. Assim como outros estudos, ${ }^{31-33,37,40,41}$ encontramos diferenças no padrão de consumo de frutas e hortaliças nos adultos de Brasília, evidenciando a necessidade de analisá-los separadamente, ao invés de considerá-los como um único grupo alimentar.

Confirmamos a hipótese do estudo deconcordância moderada e fraca para a autoavaliação do consumo de frutas e hortaliças e a avaliação técnica deste consumo. Nesse sentido, evidencia-se a falta de conhecimento dos entrevistados a respeito da quantidade que deve ser ingerida diariamente destes alimentos. Considerando-se o fato de que a maioria da amostra consome frutas e hortaliças regularmente (cinco ou mais vezes por semana), pode-se inferir que os sujeitos consideraram mais a frequência de ingestão do que a quantidade ingerida para autoavaliar o consumo atual. Outros estudos demonstram a falta de conhecimento sobre a quantidade recomendada de frutas e hortaliças. ${ }^{17,24}$ 
Atenção especial deve ser dada aos sujeitos avaliados com consumo inadequado de frutas e hortaliças e que acreditam estar consumindo porções adequadas destes alimentos. Evidências científicas demonstram que uma das principais barreiras encontradas para mudanças alimentares é a crença de que não há necessidade de mudanças, visto que o consumo atual é percebido como suficientemente saudável. ${ }^{21,22,23,24}$ Esta atitude é relatada como otimismo irreal por Raats\&Sparks ${ }^{42}$ e está presente em pessoas que se consideram abaixo da média de risco da população.

Os resultados apresentados neste estudo demonstram a necessidade do desenvolvimento de pesquisas futuras que analisem a ingestão de frutas e hortaliças separadamente e em populações de renda mais baixa, onde os fatores determinantes de consumo terão outras características. Sugere-se ainda a veiculação de medidas informativas sobre a quantidade adequada de consumo de frutas e hortaliças separadamente. Tal medida poderá esclarecer melhor a população quanto àadequação de consumo e ter repercussão favorável, especialmente nos grupos com melhor nível de escolaridade e que se consideram erroneamente adequados.

Entre as limitações do presente estudo, pode-se considerar a pequena amostra e os valores amplos dos intervalos de confiança para as razões de prevalência do modelo apresentado. No entanto, a metodologia empregada (regressão de Poisson com variância robusta) fornece uma melhor estimativa das razões de prevalência, que por sua vez representam de forma mais significativas às medidas de efeito para estudos transversais e tendem a produzir intervalos com menores amplitudes ${ }^{28}$. Além disso, deve-se destacar a importância desse trabalho pelo fato de ter sido usada uma amostra probabilística e representativa da população de interesse, que garantem a qualidade do resultado obtido. Destaca-se que a contribuição dessa pesquisa recai na identificação de características ligadas à autoavaliação e compreensão da informação veiculada pelo guia alimentar que devem ser consideradas pelos gestores, nutricionistas e educadores em nutrição e alimentação. A publicação do novo guia alimentar para a população brasileira que trás informações sobre a composição de pratos e preparações incluindo alimentos in natura como frutas e hortaliças deverão ser monitoradas para verificação de sua efetividade.

\section{Conclusão e Recomendações}

Conclui-se, a partir dos resultados do estudo, que a maioria dos indivíduos apresenta consumo de frutas e hortaliças abaixo do recomendado, não conhecem as quantidades que devem ser consumidas diariamente e o aumento dos anos de estudo (escolaridade) está associado ao consumo adequado desses alimentos. Nesses termos, recomendamos queénecessáriaa veiculação da quantidade de consumo e o tamanho da porção nas campanhas e programas de incentivo ao consumo de frutas e hortaliças para facilitar a correta percepção e escolha para o consumo. 


\section{Referências}

1. World Health Organization. Diet, nutrition and the prevention of chronic diseases. Report of a joint WHO/FAO expertconsultation. Geneva: WHO; 2003.

2. World Health Organization. Fruit and vegetable promotion initiative: report of the meeting; 25-27 August 2003. Geneva: WHO; 2003.

3. Brasil. Ministério da Saúde. Secretaria de Atenção à Saúde. Coordenação-Geral da Política de Alimentação e Nutrição. Guia alimentar para a população brasileira: promovendo a alimentação saudável. Brasília: Ministério da Saúde; 2006.

4. Brasil. Ministério da Saúde. Secretaria de Vigilância em Saúde. Secretaria de Gestão Estratégica e Participativa. Vigitel Brasil 2011: vigilância de fatores de risco e proteção para doenças crônicas por inquérito telefônico. Brasília: Ministério da Saúde; 2012.

5. Li R, Serdula M, Bland S, Mokdad A, Bowman B, Nelson D. Trends in fruit and vegetable consumption among adults in 16 US states: behavioral risk factor surveillance system, 1990-1996. Am. J. Public Health 2000; 90(5):777-81.

6. Dauchet L, Ferrières J, Arveiler D, Yarnell JW, Gey F, Ducimetière P. et al. Frequency of fruit and vegetable consumption and coronary heart disease in France and NorthernIreland: the PRIME study. Br. J. Nutr. 2004; 92(6):963-72.

7. Goss J, Grubbs L. Comparative analysis of body mass index, consumption of fruits and vegetables, smoking, and physical activity among florida residents. J. Community Health Nurs. 2005; 22(1):37-46.

8. Park SY, Murphy SP, Wilkens LR, Yamamoto JF, Sharma S, Hankin JH. et al. Dietary patterns using the food guide pyramid groups are associated with sociodemographic and life style factors: the multi ethnic cohort study. J Nutr. 2005; 135(4):843-9.

9. Neutzling MB, Rombaldi AJ, Azevedo MR, Hallal PC. Fatores asociados ao consumo de frutas, legumes e verduras em adultos de uma cidade no Sul do Brasil. Cad.Saúde Pública 2009; 25(11):2365-74.

10. Krebs-Smith SM, Kantor LS. Choose a variety of fruits and vegetables daily: understanding the complexities. J Nutr. 2001; 131(2S-1): 487S-501S.

11. Pollard J, Kirk SFL, Cade JE. Factors affecting food choice in relation to fruit and vegetable intake: a review. Nutr. Res. Rev. 2002; 15(2):373-87.

12. Figueiredo ICR, Jaime PC, Monteiro CA. Fatores asociados ao consumo de frutas, legumes e verduras em adultos da cidade de São Paulo. Rev. Saúde Pública 2008; 42(5):777-85.

13. Kant AK, Graubarg BI. Energy density of diets reported by American adults: association with food group intake, nutrient intake, and body weight. Int J Obesity 2005; 29(8):950-6.

14. Pullen CH, Walker SN, Hageman PA, Boeckner LS, Oberdorfer MK. Differences in eating and activity markers among normal weight, overweight, and obese rural women. Womens Health Issues 2005; 15(5):209-15.

15. Pollard J, Greenwood D, Kirk S, Cade J. Motivations for fruit and vegetable consumption in the UK Women's Cohort Study. Public Health Nutr. 2002; 5(3):479-86. 
16. Satia JA, Kristal AR, Curry S, Trudeau E. Motivations for health ful dietary change. Public Health Nutr. 2001; 4(5):953-9.

17. Watters JL, Satia JA, Galanko JA. Associations of psycho social factors with fruit and vegetable among African-Americans. Public Health Nutr. 2007; 10(7):701-11.

18. Yeh M, Ickes SB, Lowenstein LM, Shuval K, Ammerman AS, Farris R. Understanding barriers and facilitators of fruit and vegetable consumption among a diverse multi-ethnic population in the USA. Health Promot. Int. 2008; 23(1):42-50.

19. Silva CL, Costa THM. Barreiras e Facilitadores do consumo de frutas e hortaliças em adultos de Brasília. Sci. Med. 2013; 23(2):68-74.

20. World Health Organization. Fruit and vegetables for health. Report of a joint FAO/WHO workshop, 1-3 september, 2004; Kobe, Japan. Geneva: WHO; 2004.

21. Kearney M, Gibney MJ, Martinez JA, Almeida MD, Friebe D, Zunft HJ et al. Perceived need to alter eating habits amongre presentative samples of adults from all member states of the European Union. Eur. J. Clin. Nutr. 1997; 51(suppl 2): S30-5.

22. Kearney JM, McElhorne S. Perceived barriers in trying to eathealthier: results of pan-EU consumer attitudinal survey. Br. J. Nutr. 1999; 81(Suppl 2):S133-7.

23. Biloukha OO, Utermohlen V. Health yeating in Ukraine: attitudes, barriers and information sources. Public Health Nutr. 2001; 4(2):207-15.

24. Toral N, Slater B, Cintra IP, Fisberg M. Comportamento alimentar de adolescentes em relação ao consumo de frutas e verduras. Rev. Nutr. 2006; 19(3):331-40.

25. Thomaz PMD, Costa THM, Silva EF, Hallal PC. Fatores associados à atividade física em adultos, Brasília, DF. Rev. Saúde Pública 2010; 44(5):894-900.

26. Thomaz PMD, Silva EF, Costa THM. Validade de peso, altura e índice de massa corporal autorreferidos na população adulta de Brasília. Rev. Bras. Epidemiol. 2013; 16(1):157-169.

27. Lipschitz DA. Screening for nutritional status in the elderly. Prim. Care 1994; 21(1):55-67.

28. Coutinho LMS, Scazufca M, Menezes PR. Métodos para estimar razão de prevalencia em estudos de corte transversal. Rev. Saúde Pública 2008; 42(6):992-8.

29. Associação Brasileira das Empresas de Pesquisa. Critério classificação Brasil, 2003 [Internet]. São Paulo: ABEP. [Acesso em: 12 dec. 2010]. Disponível em: http://www.abep.org/criterio-brasil

30. Moyses S, Nieto FJ. Epidemiology beyond the basis. Maryland: Aspen; 2000.

31. Ashfield-Watt PAL, Welch AA, Day NE, Bingham SA. Is five -a- day an effectiveway of increasing fruit and vegetable intakes? Public Health Nutr. 2004; 7(2):257-61.

32. Naska A, Vasdekis VGS, Trichopoulou A, Friel S, Leonhäuser IU, Moreiras O. et al. Fruit and vegetable availability among ten European countries: how doesit compare withthe 'five-a-day' recommendation? Br. J. Nutr. 2000; 84:549-56. 
33. Brasil. Ministério da Saúde. Secretaria de Vigilância em Saúde. Secretaria de Gestão Estratégica e Participativa. Vigitel Brasil 2007: vigilância de fatores de risco e proteção para doenças crônicas por inquérito telefônico. Brasília: Ministério da Saúde; 2008.

34. Jorge MIE, Martins IS, Araújo EAC. Diferenciais socioeconômicos e comportamentais no consumo de hortaliças e frutas em mulheres residentes em município da região metropolitana de São Paulo. Rev. Nutr. 2008; 21(6):695-703.

35. Peixoto MRG, Monego ET, Alexandre VP, Souza RGM, Moura EC. Monitoramento por entrevistas telefônicas de fatores de risco para doenças crônicas: experiência de Goiânia, Goiás, Brasil. Cad. Saúde Pública 2008; 24(6):1323-33.

36. Girois SB, Kumanyika SK, Morabia A, Mauger E. A comparison of knowledge and attitudes about diet and health among 35- to 75-year-old adults in the United States and Geneva, Switzerland. Am. J. Public Health 2001; 91(3):418-24.

37. Morimoto JM, Latorre MRDO, César CLG, Carandina L, Barros MBA, Goldbaum M, et al. Fatores associados à qualidade da dieta de adultos residentes na Região Metropolitana de São Paulo, Brasil, 2002. Cad. Saúde Pública. 2008; 24(1):169-78.

38. Rautiainen S, Wang L, Lee IM, Manson J, Buring JE, Sesso H. Higher intake of fruit, but not vegetables or fiber, at baseline is associated with lower risk of becoming overweight or obese in middle-aged and older women of normal bmi at baseline. J. Nutr. 2015; 145(5):960-8.

39. te Velde SJ, Twisk JW, Brug J. Tracking of fruit and vegetable consumption from adolescence into adulthood and its longitudinal association with overweight. Br. J. Nutr. 2007; 98(2):431-8

40. Bodor JN, Rose D, Farley TA, Swalm C, Scott SK. Neighbourhood fruit and vegetable availability and consumption: the role of small food stores in an urban environment. Public Health Nutr. 2007; 11 (4):413-20.

41. Marinho MCS, Hamann EM, Lima ACCF. Práticas e mudanças no comportamento alimentar da população de Brasília, Distrito Federal, Brasil. Rev. Bras. Saúde Matern. Infant. 2007; 7(3):251-6.

42. Raats MM, Sparks P. Unrealistic optimism about diet-related risks: implications for interventions. Proc. Nutr Soc. 1995; 54 (3):737-45.

Recebido: $11 / 6 / 2015$

Revisado: $16 / 9 / 2015$

Aprovado: 05/10/2015 